\title{
Thermal and Mechanical Modeling of Fluid and Heat Flow in a Porous Metal Using Neural Networks for Application as TPS in Space Vehicles
}

\author{
Prabhu. S. M.*, Abbas Soundarajan Mohadeen \\ Jhon Bosco college of Engineering Tiruvallur 631203 \\ Mamallan inst of technology Sriperumbudur \\ E-mail:prabhusm2005@gmail.com,mamalan_princi@hotmail.com \\ Received May 9, 2011; revised May 31, 2011; accepted June 5, 2011
}

\begin{abstract}
This paper contains novel model using feedback neural networks for a work piece temperature prediction. The heat and mass transfer in a porous metal workpiece which is heated by a fire gun is studied. The heat flux distribution is determined by thermocouple connected on the workpiece at definite distances. The gun work piece distance were also change and the temperature distribution and heat flux were determined. The permeability's were in range of $0.01-0.15$. The ANN model parameters of the result output were simulated using the ANN parameters the simulation was done using MATLAB $6.0^{\circledR}$ Neural Network Toolbox.
\end{abstract}

Keywords: Neyral Network, Porous Media, Prous Passages

\section{Introduction}

The heat and mass transfer in a porous metal workpiece which is heated by a fire gun is studied. The heat flux distribution in a workpiece due to effect of thermal spray nozzle (uniform external heat source) causing high radiation conduction and convection transfer in the workpiece. The flame gun was kept at various distance and the effect of the source studied for the radiation and convection. This work mainly deals with the studies on heat flux and temperature prediction in a metal workpiece subjected to firing gun (fired using mixture of oxygen and acetylene gas) generally used for gas cutting and welding operations

Studies on Flow and transport at interface between a porous medium and clear fluid have been studied widely. Fauchius, Vandelle and others [1] studied the mechanisms and models for Thermal spraying. Sampath and Jiang [2] discussed the procedures for computing design parameters in Substrate temperature spray coatings. Pop [3] did studies on modeling of heat flow in a porous cavity. Lai [4] studied the effect of Non-Darcy Convection in the free surface of a porous media. In this paper a gener- alized treatment of convection force and the effect of free surface hydrodynamics on the heat transfer is dealt with. A novel model incorporating computation of local and average heat flux has been discussed. Ras $[5,6]$ did studies on the free surface convection while others including Lai [4] studied the Forced convection on the surface of porous layer. Pop and Postelnicu [7] discussed on the effect of heat generation effect on the layers in porous media.

Flow and transport at interface between a porous medium and clear fluid is of importance in heat transfer in porous enclosures and extended surfaces. The mechanisms that contribute to the enhanced heat transfer include heat conduction in the metal foam matrix (whose conductivity is always higher by several orders of magnitude .The well known Darcy's law is based on a balance between the present gradient and the viscous forces and breaks down for high velocities when inertia terms are no longer negligible. Here we present numerical and experimental results for buoyancy induced flow in a high porosity metal foam

The Associative Neural network (ASNN) is an extension of the ANN that goes beyond a simple/ weighted average of different models. Associative Neural network represents a combination of an ensemble of feed-forward neural networks and the $k$-nearest neighbor technique. It uses the correlation between ensemble responses as a measure of distance amid the analyzed cases for the ANN. This corrects the bias of the neural 
network ensemble. An associative neural network has a memory that can coincide with the training set. If new data becomes available, the network instantly improves its predictive ability and provides data approximation (self-learn the data) without a need to retrain the ensemble. Another important feature of ASNN is the possibility to interpret neural network results by analysis of correlations between data cases in the space of models. BAM (binary associative memory) is the recurrent memory achieved by the exemplar on being trained by standard inputs prior to its deployment for modeling and simulation applications

\section{Experimental Methods:}

The heat flux distribution is determined by thermocouple connected on the workpiece at definite distances. The gun work piece distance were also changed and the temperature distribution and heat flux were determined. $\mathrm{Cu}-\mathrm{Ni}$ Thermocouples were attached on the surface of the workpiece. The flame was generated by acetylene and oxygen gasses as in gas welding process, the flame was kept at distances from $5 \mathrm{~cm}$ to $20 \mathrm{~cm}$ and the heat flux distribution studied.

During a typical experimental run the powers were varied to achieve different base late temperature and hence Rayleigh numbers. Due to temperature constraints the parameters of the heat input were restricted to maximum base plate temperature of $80 * \mathrm{c}$ a metal foam sample heated from above .

The foam sample is saturated with \& surrounded by a fluid, which extends a distance $s_{1}$ in $x$ direction and $s_{2}$ in y direction.

The steady two dimensional equations for the fluid saturated porous medium \& for the clear fluid region outside the metal foam are written separately as shown below.

The Flow in a Square Pore cavity is modeled using the continuity and energy equations using a square computational grid with velocity and temperature boundaries.

The continuity and energy equations are given by

$$
\begin{gathered}
\frac{\partial u}{\partial r}+\frac{\partial v}{\partial \theta}=0 \\
\frac{1}{\varepsilon^{2}} \frac{u \partial u}{\partial r}+\frac{v \partial v}{\partial r}=a\left[\frac{\partial^{2} u}{\partial r^{2}}+\frac{\partial}{\partial \theta^{2}}+\rho \frac{\partial T}{\partial \theta}\right]
\end{gathered}
$$

where volumetric heat sources $(r, y, z)$ represents the contribution of frictional heating. The parameters $\rho C_{p}$ \& $k_{e}$ may depend on $y \quad \& \quad z$ but remain independent of $r$. More importantly the contribution of axial conduction deferred to the subsequent is neglected, hence Equation (4) reduces

$$
\begin{aligned}
& u \frac{\partial u}{\partial r}+v \frac{\partial v}{\partial r} a\left[\frac{\partial^{2} u}{\partial r^{2}}+\frac{\partial^{2} v}{\partial \theta^{2}}\right]+\frac{\mu}{k \rho c_{p}}\left(u^{2}+v^{2}\right) \\
& u \frac{\partial T}{\partial r}+v \frac{\partial T}{\partial r} \\
&=\frac{\partial}{\partial r}\left[\lambda \frac{\partial T}{\partial r}\right]+\frac{\partial}{\partial \theta}\left[\lambda \frac{\partial T}{\partial \theta}\right]+(1-\varepsilon) Q_{0}
\end{aligned}
$$

where the Heat generation number $Q_{d}$ is given by

$$
Q_{0}=\frac{L_{r e f}^{2}}{\alpha_{f}} \frac{q^{\prime \prime}}{\rho_{f} C_{p f}\left(T_{h}-T_{c}\right)}
$$

The governing equations are scaled on basis of fluid Raleigh number as in the case of viscous fluid (independent of permeability) instead of modified Raleigh's number. The scaling is introduced to study the effective change in values of individual matrix \& fluid parameters (6). Finite element method is used for prediction of parameters. A suitable grid scheme with iso-parametric, quadrilateral elements is used for stability of numerical solution, all the elements are containing 8 nodes, one at each corner and one at midpoint of each of the sides. All nodes are given velocity \& temperature boundaries and corners of the grid pressure boundaries. This is an accepted practice given by Taylor (2). For depicting the variation in pressure by the shape function $M_{i}$ of one order less than shape function $N_{i}$ defined for velocities and temperature.

\subsection{Direct Problem:}

$$
\begin{gathered}
\rho C_{p} \frac{\partial T}{\partial r}=k \frac{\partial}{\partial r} r \frac{\partial T}{\partial r}+\frac{\partial^{2} T}{\partial z} \\
-\frac{k \partial T}{\partial z}=q(r, t) \quad \mathrm{t}>0 \quad r \in(0, R) \quad z=H \\
\frac{\partial T}{\partial n}=0 z=0 r=[0 . R] \\
T=T_{0} \quad t=0 \quad \text { at } r \in[0, R]
\end{gathered}
$$

\subsection{Sensitivity Problem}

$$
\begin{gathered}
\rho C_{p} \frac{\partial \delta T}{\partial r}=k \frac{\partial}{\partial r} r \frac{\partial \delta T}{\partial r}+\frac{\partial^{2} \delta T}{\partial z} \\
\frac{k \partial T}{\partial z}=q(r, t) \quad t>0 \quad r \in(0, R) z=H \\
\frac{\partial T}{\partial n}=0, z=0, r=[0 . R]
\end{gathered}
$$




\subsection{Adjoint Problem}

$$
J(q)=\sum \sum r\left[T\left(r_{m s}, z_{m s}, t ; q\right)-Y_{m s}(t)\right]^{2}
$$

where $T\left(r_{m s}, z_{m s}, t, q\right)$ is the temperature calculated at the measuring points (rms) through the direct problem Equations 5 \& (5a)-(c), $\mathrm{Yms}(t)$ is measured temperature and the subscript ms denotes the thermocouple number.

The adjoint problem is obtained by introducing the Lagrange multiplier $\psi(r, z, t)$ into the direct problem equations and by integrating over the spatial and then over the temporal domain as follows.

For the region $r \in(0, R), z \in(0, H)$

$$
\begin{gathered}
\rho C_{p} \frac{\partial T}{\partial t}=k \frac{\partial}{\partial t}\left[\frac{r \partial \psi}{\partial r}+\frac{\partial^{3} \psi}{\partial z}\right] \\
=[T(r, z, t, q)-Y(t)] \frac{\delta\left(r-r_{w}\right)}{\partial\left(z-z_{m s}\right)} \text { at } \mathrm{t}>0 \\
\frac{\partial \psi}{\partial n}=0, z=0, r=[0 . R], \mathrm{z}=0, H \\
\psi=0 \quad t=t_{f} \quad r \in=[0 . R], z=0 H
\end{gathered}
$$

where $\delta(\cdots)$ is the Dirac delta function. It is required for solving the adjoint equations with the end condition at time $t=t_{f}$, one should first proceed with variable change $\tau=t-t_{f}$, The adjoint problem becomes an initial value problem in $\tau$ via the transformation.

\section{Step size and Gradient}

The iterative equation for determining $q(r, z, t)$ can be given by

$$
q^{n+1}(r, t)=q^{n+1}(r, t)-\beta P^{n}(r, t)
$$

where the superscript $n$ denotes the iteration number and $\beta^{n}$ is the search stepsize defined by

$$
\beta^{n}=\frac{\int_{0}^{H} \int_{0}^{H}\left[\delta T^{n}\left(r_{m z}, z_{m s}, t ; q\right)-Y_{m s}(t)\right] d r d t}{\int_{0}^{H} \int_{0}^{R} r \delta T^{n}\left(r_{m z}, z_{m s}, t ; q\right)^{2} d r d t}
$$

and the descending direction $P^{n}$ is estimated by

$$
p^{n}(r, z, t)=\psi^{n}+\gamma^{n} p^{n-1}(r, z, t)
$$

Where $n=1, \gamma^{1}=0$. General definition of $\gamma$ is given by

$$
\frac{\gamma^{n}=\left[\psi\left(q^{n}\right), \psi\left(q^{n}\right)-\psi\left(q^{n-1}\right)\right]}{\left\|\psi\left(q^{n-1}\right)\right\|^{2}}
$$

where $\|\cdot\|$ represents the norm and (.,.) the scalar product.

\begin{tabular}{|c|c|c|c|c|}
\hline Category & Parameter & $\begin{array}{l}\text { Lower } \\
\text { limit }\end{array}$ & $\begin{array}{l}\text { Upper } \\
\text { limit }\end{array}$ & $\begin{array}{l}\text { Neuron } \\
\text { number }\end{array}$ \\
\hline \multirow{6}{*}{$\begin{array}{l}\text { Input pat- } \\
\text { tern }\end{array}$} & $\begin{array}{l}\text { D gun workpiece dis- } \\
\text { tance }\end{array}$ & 30 & 100 & 1 \\
\hline & $t$-preheating time $s$ & 0 & 10 & 1 \\
\hline & $\begin{array}{c}Y_{1} \text { Firstthermocouple } \\
\text { temperature }\end{array}$ & 10 & 60 & 3 \\
\hline & $\begin{array}{c}Y_{2} \text { secondthermocouple } \\
\text { temperature }\end{array}$ & 10 & 65 & 2 \\
\hline & $\begin{array}{c}Y_{3} \text { thirdthermocouple } \\
\text { temperature }\end{array}$ & 10 & 60 & 5 \\
\hline & radial position mm & 0 & 50 & 4 \\
\hline $\begin{array}{l}\text { Output } \\
\text { pattern }\end{array}$ & $q$-heat flux Mw/sqm & 0 & 1.2 & 1 \\
\hline
\end{tabular}

\section{Time stepsize and algorithm stop criterion}

The stopping criterion is satisfied when the Fourier
Table 1. Properties of workpiece (Cu).
Thermal conductivity $k=396 \mathrm{w} / \mathrm{m} \cdot \mathrm{c}$

$C_{p} 389 \mathrm{~J} / \mathrm{kg} \cdot \mathrm{c}$

Density $=8900 \mathrm{~kg} / \mathrm{m}^{3}$

Table 1a. Artificial Neural Network (ANN) variable description.

number $\left(F_{o}\right)$ satisfies the condition

$$
F_{o}=\frac{k}{\rho C_{p}} \cdot \frac{\Delta t}{H_{t p}}>0.05
$$

where $F_{o}=0.028, \Delta$ is the time step, and $H_{t p}$ is the location of thermocouples to the surface in front of the flame ,according to parameters listed in Table 1.

Since the Fourier number satisfies Equation (17), the increase of measuring errors is weak and few obvious noisy signals can be captured. In this study, $\Delta t=0.5 \mathrm{~s}$ has been considered for the time step according to the limit of data acquisition system. If the problem contains no noisy signals, the general stopping criterion condition is expressed by

$$
J(q)<\varepsilon
$$

Where $\psi$ is specified stopping criterion. But the measurement precision of data $\psi$ acquisition is about $10^{-3}$. Hence it should not be reasonable to expect the functional Equation (9) to be equal to zero. A small value of $\varepsilon=0.002$ is chosen for above criterion

\section{Algorithm for CGM method}

The algorithm for Conjugate gradient method (CGM) is given below.

1) Select an initial guess $q^{n}(r, t)$. generally equal to zero.

2) Calculate the direct problem, Equations (1)-(4), obtain the solution $T\left(r_{m s}, z_{m s}, t ; q^{n}\right)$.

3) Decide if the stopping criterion Equation (18) is satisfied. If yes go to step (6), otherwise continue.

4) Solve the adjoint problem. Equations (10)-(12) [note: $\tau=\left(\mathrm{t}_{1}-t\right)$ in place of $\mathrm{t}$ ], calculate the conjugate 
Table 2. ANN output results of parameters.

\begin{tabular}{|c|c|c|c|c|c|c|c|c|c|c|c|}
\hline Time & $t$ secs & $y_{r}$ & $\boldsymbol{y}_{p}$ & $\boldsymbol{E}_{t ;}$ & $I_{k l}$ & $D \mathbf{m}$ & $\begin{array}{ll}Y_{1} & \mathbf{m}\end{array}$ & $Y_{2} \mathbf{m}$ & $\begin{array}{ll}Y_{3} & \mathbf{m}\end{array}$ & Radial posn heat flux & $q \mathrm{MW} / \mathrm{sqm}$ \\
\hline & 8 & 0.87 & 0.54 & 0.05 & 0.51 & 0.056 & 0.028 & 0.039 & 0.06 & 20 & 0.7 \\
\hline & 9 & 0.92 & 0.63 & 0.07 & 0.73 & 0.082 & 0.035 & 0.054 & 0.064 & 22 & 0.81 \\
\hline & 10 & 1.01 & 0.78 & 0.095 & 0.81 & 0.093 & 0.055 & 0.07 & 0.073 & 26 & 0.98 \\
\hline & 12 & 1.21 & 0.91 & 0.11 & 0.96 & 0.098 & 0.07 & 0.085 & 0.082 & 29 & 1.03 \\
\hline
\end{tabular}

Table 3. Computation of flux parameters using CGM.

\begin{tabular}{|c|c|c|c|c|c|c|c|c|c|c|c|}
\hline Time $t$ secs & $n(\mathbf{c m})$ & $P^{n}(r, z, t)$ & $\cdot q^{n+1}(r, t)$ & $T_{f}$ & $T$ & $\tau=T_{f}-T$ & $E_{o}^{t^{\prime}}$ & $\gamma$ & $\cdot q^{\prime}$ & $F_{o}$ & $Q_{d}$ \\
\hline & 2 & 1.83 & 0.628 & 340 & 280 & 60 & 0.15 & 1.5 & 2.43 & 0.185 & 0.003 \\
\hline \multirow[t]{3}{*}{2} & 4 & 1.84 & 0.651 & 347 & 284 & 63 & 0.173 & 1.48 & 2.45 & 0.21 & 0.0034 \\
\hline & 6 & 1.91 & 0.731 & 378 & 293 & 68 & 0.18 & 1.54 & 2.63 & 0.27 & 0.0041 \\
\hline & 2 & 1.41 & 0.78 & 390 & 297 & 72 & 0.21 & 1.64 & 2.71 & 0.32 & 0.0046 \\
\hline \multirow[t]{3}{*}{4} & 4 & 1.63 & 0.82 & 410 & 302 & 78 & 0.28 & 1.81 & 2.91 & 0.45 & 0.0047 \\
\hline & 6 & 1.73 & 0.87 & 412 & 308 & 81 & 0.32 & 1.92 & 3.12 & 0.51 & 0.0049 \\
\hline & 2 & 1.45 & 0.81 & 402 & 304 & 74 & 0.23 & 1.71 & 2.8 & 0.41 & 0.005 \\
\hline \multirow[t]{3}{*}{6} & 4 & 1.71 & 0.92 & 408 & 307 & 76.3 & 0.28 & 1.81 & 2.9 & 0.43 & 0.0051 \\
\hline & 6 & 1.81 & 1.02 & 415 & 308 & 77.4 & 0.41 & 1.93 & 3.2 & 0.48 & 0.0053 \\
\hline & 2 & 1.84 & 1.08 & 418 & 312 & 79 & 0.48 & 2.01 & 3.43 & 0.52 & 0.0054 \\
\hline \multirow[t]{2}{*}{8} & 4 & 1.86 & 1.13 & 421 & 315 & 81 & 0.51 & 2.11 & 3.51 & 0.58 & 0.0056 \\
\hline & 6 & 1.91 & 1.31 & 428 & 321 & 85 & 0.61 & 2.33 & 3.81 & 0.7 & 0.0058 \\
\hline
\end{tabular}

coefficient $\gamma^{n}$, Equation (19) and the direction of descendent $p^{n}$ Equation (18).

5) Solve the sensitivity problem Equations (5)-(8), calculate the stepsize $\beta^{n}$ from the nth to the $(n+1)$ st iteration, Equation (17) and calculate a new vector $q^{n+1}$. Go to step 2.

6) The iteration is terminated.

\section{A Neural Computational Procedure}

The heat flux calculated by solving the inverse problem was correlated to the workpiece temperature and other parameters with the aid of an artificial neural network (ANN). Such a structure considers three main categories.

1) The processing and the experiment design parameters with aid of an artificial neural network (ANN). Such a structure considers following types.

2) The processing and experiment design parameter as an input (1) pattern. These were the gun workpiece distance, the preheating time, the thermocouple temperatures and radial position.

3) The heat flux as an output (O) unit calculated using CGM.

4) An intermediate structure called hidden layers, encoding the correlations between I/O patterns.

Each of the ANN types is defined by a set of neurons playing role of simple processing elements as in Table 1a. A neuron has ability to receive a sum of numbers from other neurons and to emit a signal number toward other neurons according to

$$
I_{k 1}=W_{i j k 1} O_{i j}
$$

where $I_{k 1}$ is input of neuron 1 from layer $k, O_{i j}$ is output of neuron $\mathrm{j}$ from layer; $W_{i j k 1}$ is called the weight relating neuron $\mathrm{j}$ and neuron 1 and this corresponds to neuron strength

$$
q^{n+1}(r, t)=q^{n}(r, t)-\beta P^{n}(r, t)=2.35 \times 1.45=0.628
$$

from (16)

$$
\begin{gathered}
\frac{\gamma^{n}=\left[\psi\left(q^{n}\right), \psi\left(q^{n}\right)-\psi\left(q^{n-1}\right)\right]}{\left\|\psi\left(q^{n-1}\right)\right\|^{2}}=\frac{1.2-0.73}{1.43^{2}}=0.243 \\
F_{0}=\frac{k}{\rho C_{p}} \frac{\Delta t}{H_{t p}}>0.05=\alpha \Delta t / H_{t p} \\
=\frac{396}{8900 \times 389} \cdot \frac{1.5}{0.0021}=0.885>0.05
\end{gathered}
$$

from (14)

$$
\begin{aligned}
P^{n}(r, z, t) & =\psi^{n}+\gamma^{n} P^{n-1}(r, z, t) \\
& =1.2+1.5 \times(0.43)=1.83
\end{aligned}
$$

from (4a) we have 


$$
\begin{gathered}
Q_{d}=\frac{L_{r e f}{ }^{2} q^{\prime \prime \prime}}{\alpha_{f} \rho_{f} C_{p f}\left(T_{h}-T_{c}\right)} \\
=\frac{0.85^{2} \times 154}{0.00011 \times 8900 \times 389(260-118)}=0.0028 \mathrm{~kJ} / \mathrm{kg} \cdot \mathrm{s}
\end{gathered}
$$

\section{A Neural Network Model Formulation}

The CGM solution is validated by comparing the temperatures at measuring points recorded by data acquisition unit with those of one step direct problem resolution with the convergent solution regarded as the known boundary conditions. The heat flux profiles were compared with simulated and experimental Values. For the ANN simulation computation was done using Conjugate Gradient Method (CGM).

Different iteration levels were considered from $10^{3}$ to $2.5 \times 10^{5}$. The best compromise was obtained for 1500 cyclessince over-fitting is avoided .The best compromise was at $8 \times 20$ Different ANN configurations with the ANN convergence property was validated as the ratio of classification was equal to $100 \%$ at tend of iterative process. Different ANN configurations were examined introducing neuron penalty "permitting to add or remove neurons depending on the training or the test error evolutions. The optimization procedure permitted us to obtain a two layer structure as in Figure. The first hidden layer contained 13 neurons and the second one contained 8 neu-

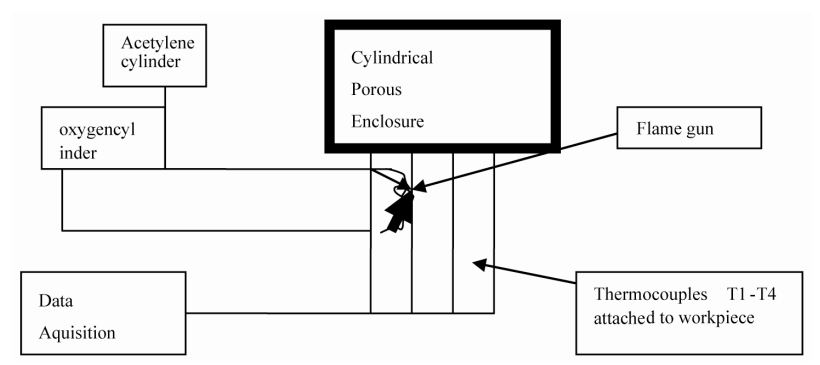

Figure 1. Metal foam sample experiments experimental setup of gas welding.

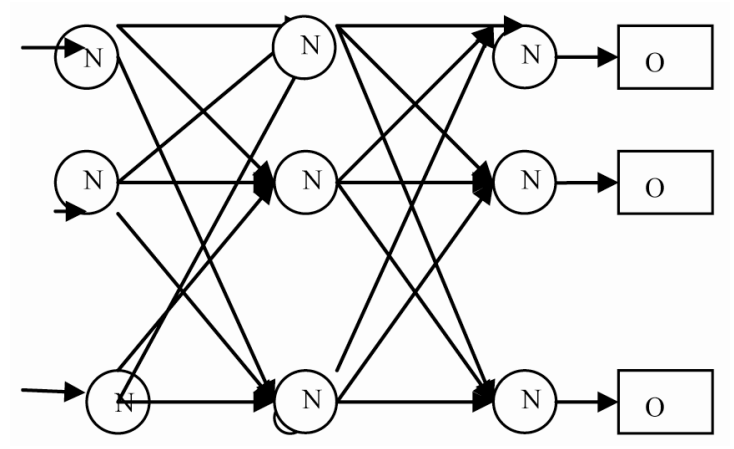

Figure 2. Map of two layer BAM neural network for the problem model. rons. Such structures are known to be sufficient to encode nonlinear correlations. At the end of iterative process the average and maximum training errors were $7 \times 10^{-3}$ and $2.5 \times 10^{-2}$ respectively. Figure shows the instantaneous heat flux profiles determined by the CGM and the ANN for the gun workpiece distance $30 \mathrm{~mm}$. Along the radial direction the flux strength decreases rapidly. In the proximity of $z$ axis the flux distribution appears singular because of unavailability of temperature information between $r=0$ and $r=20 \mathrm{~mm}$. The same phenomena can also be found between $r=20$ and $40 \mathrm{~mm}$ for gun workpiece distance of $60 \mathrm{~mm}$.There is an inflexion point away from which axis; this means that the flux becomes more and more concentrated i.e decrease of effective preheating. Moreever the maximal flux heat for $30 \mathrm{~mm}$ reaches $1.1 \mathrm{MW} / \mathrm{m}^{2}$ which is four times larger than $90 \mathrm{~mm}$.

The maximum of heat flux calculated corresponds to the heat flux experienced by the workpiece at the $Z$ position corresponding to the geometric axis of the flame gun. Thus this value is expected to be the largest one at the surface of the workpiece at the $Z$ position corresponding to geometric axis of the flame gun.

A Two layer BAM (Binary Associative memory) Hopfield network for the flame gun temperature \& flux estimation.

It can also be observed that the heat flux profiles estimated by the ANN are very similar to those of the CGM.

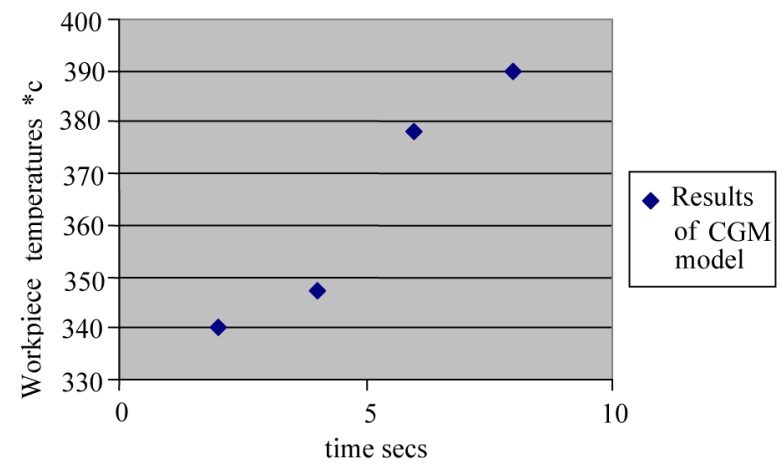

Figure 3. Predicted temperature profiles.

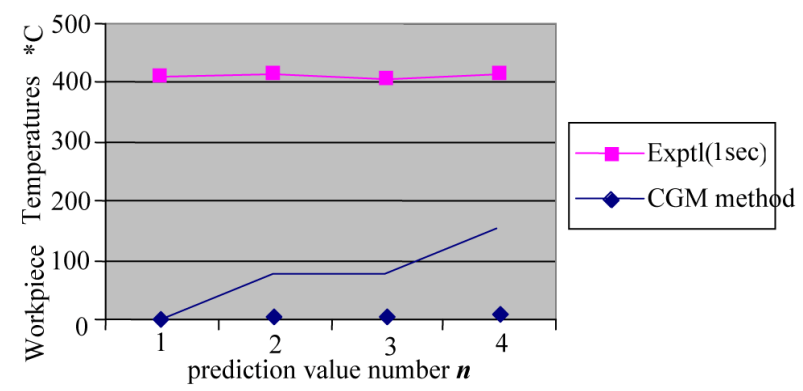

Figure 4. Temperature prediction at different points. 
The peak values at the $Z$ axis for workpiece distances 30, 60 and $90 \mathrm{~mm}$ are about 1.1.0.8 and 0.3 Mw/sqm for the two solutions.

To compare the $q-r$ profiles calculated by CGM and ANN methodologies an average error o the preheating time was considered. Table 3 shows the errors obtained for different gun workpiece distances. It appears that the average error depends on mesh density is not high enough to allow the ANN structure to finally describe the exponential decay of the heat flux mechanism

ANN has the ability to predict property evolution for intermediate and limiting parameter values. Two examples for such properties are presented through the consideration of optimized ANN structure where the $q-r$ curves were built for the gun workpiece distances of $45 \mathrm{~mm}$ and $75 \mathrm{~m}$. These two distances belong to the parameter space defined for the ANN structure. As at the ANN input, thermocouple temperatures are needed to build the $q-r$ curve; these were calculated using the following linear relationship

$$
\left\{\begin{array}{l}
Y_{m s t}^{45}=\frac{Y_{m s t}^{30}+Y_{m s t}^{60}}{2} \\
Y_{m s t}^{75}=\frac{Y_{m s t}^{60}+Y_{m s t}^{90}}{2} \quad m s=1 . .5 ; \quad t=1 . .7
\end{array}\right.
$$

where the subscripts $m s$ and $t$ are relative to the th-

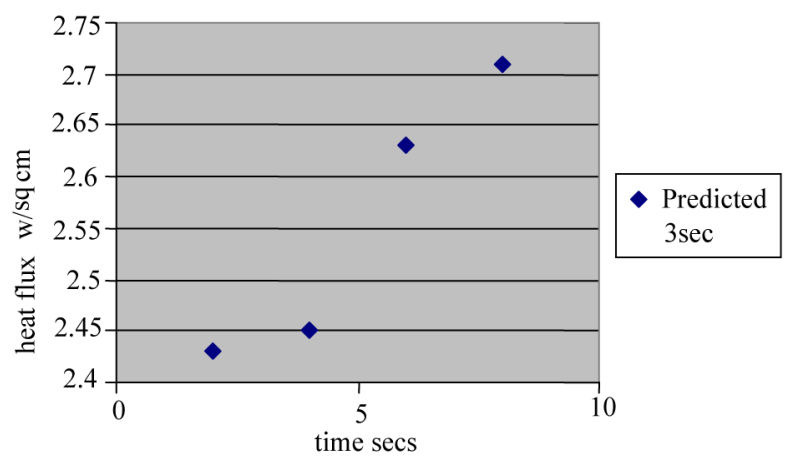

Figure 5. Heat flux profiles workpiece dist $60 \mathrm{~mm}$.

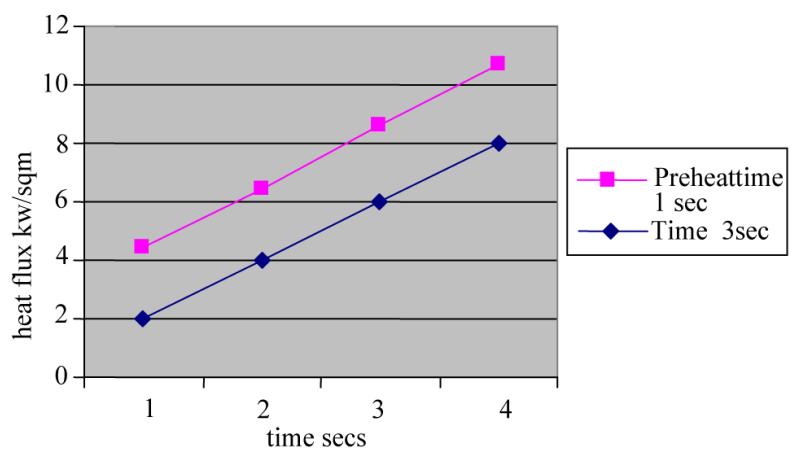

Figure 6. Heat flux profile (workpiece distance $90 \mathrm{~mm}$ )
Table 4. Artificial Neural Network (ANN)variable description.

\begin{tabular}{ccccc}
\hline Category & Parameter & $\begin{array}{c}\text { Lower } \\
\text { limit }\end{array}$ & $\begin{array}{c}\text { Upper } \\
\text { limit }\end{array}$ & $\begin{array}{c}\text { Neuron } \\
\text { number }\end{array}$ \\
\hline & D gun workpiece dis- & 30 & 100 & 1 \\
tance & $t$-preheating time $s$ & 0 & 10 & 1 \\
$\begin{array}{c}\text { Input pat- } \\
\text { tern }\end{array}$ & $\begin{array}{c}\text { temperature } \\
Y_{2} \text { secondthermocouple } \\
\text { temperature }\end{array}$ & 10 & 60 & \\
& $Y_{3}$ thirdthermocouple \\
temperature & 10 & 60 & 5 \\
Output & radial position mm \\
pattern & $q$-heat flux Mw/sqm & 0 & 50 & 1 \\
\hline
\end{tabular}

ermocouple position and the preheating time, and the superscript is relative to gun workpiece distance.

Interpolation of thermocouple temperatures are introduced in order to predict realistic behavior related to intermediate workpiece distances. Indeed as the ANN structure learns from the database, any set of thermocouple temperatures can be introduced as input parameter.

The calculated heat-flux profiles represent actual intermediate evolutions when comparing maximum strengths $(r=0)$. Whatever the gun workpiece distance the heat flux was found to increase with the preheating

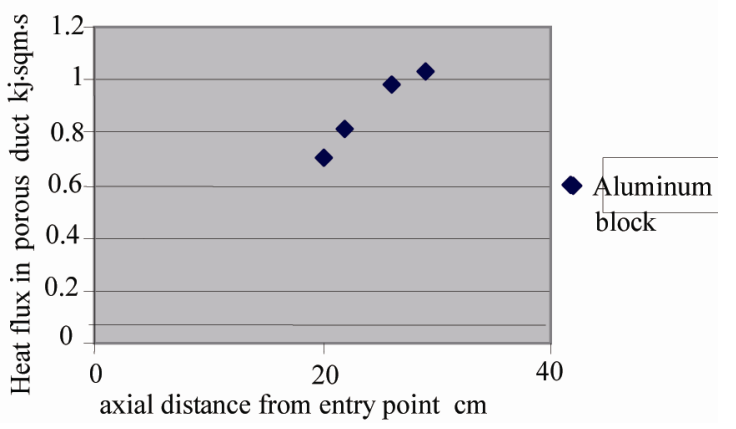

Figure 7. Experimental values of heat flux profile using model equations.

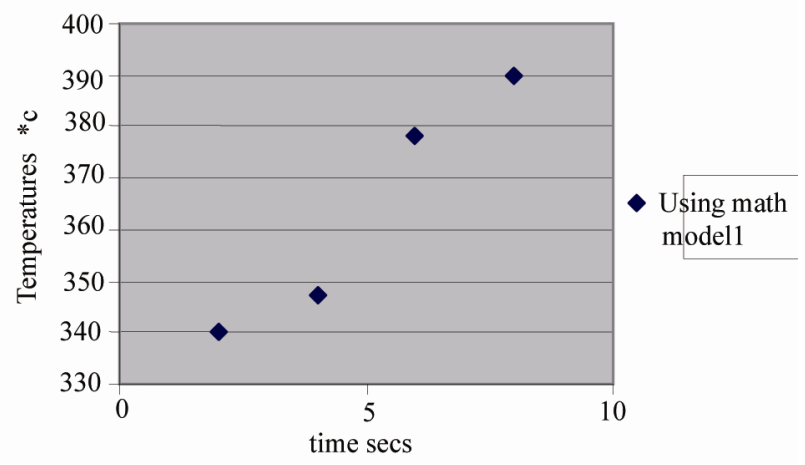

Figure 8. Predicted temperature profiles. 
time for a given radial position ad to decrease with increase of radial distance for a given preheating Time. The inflection point for the intermediate gun- work-piece distance $45 \mathrm{~mm}$ is located at $30 \mathrm{~m}$ which is the intermediate value between the cases of gun work- piece distances of $30 \mathrm{~m}$ and $60 \mathrm{~mm}$.

\section{Results and Discussion}

Figure 2 shows the map of Two layer BAM neural network for the problem model which was used for our computation The Figure 3 shows the plot of predicted temperature profiles at $3.5 \mathrm{kj} \cdot \mathrm{sqcm}$. Next the Figure 4. depicts the temperature prediction at different points at a input value of the heat flux being $6 \mathrm{~kJ} / \mathrm{cmsq}$. The temperatures gradually decreased form the heated end of the workpiece. The Figure 5 shows the heat flux profile (at a workpiece distance $60 \mathrm{~mm} /$, The heat flux is initially steady and decreases over the middle of the workpiece, This is attributed to the accelerated heat dissipation on the surface of the porous metal workpiece, Finally Figure 6 shows the heat flux profile. Here the workpiece distance was kept at $90 \mathrm{~mm}$ from the laser gun used for heating it. Figure 7 gives the depiction of Experimental values of heat flux profile using model equations .These data correlated with the results of the neural network model whose results are given in Figures 3-6 and Tables 4-6. Figure 8 shows the .predicted temperature profiles in the workpiece computed using the CGM method. Table 1 gives the .Properties of workpiece (Cu). Table 2 gives the ANN model parameters of the result output simulated using the ANN parameters the simulation was done using MATLAB 6.0 ${ }^{\circledR}$ Neural Network Toolbox. Table 6 Computation of flux parameters using CGM. Table 5 deicts. ANN output results of parameters. Table 4 Artificial Neural Network (ANN) variable description. Table 1a gives the description of parameters of an Artificial Neural Network (ANN) variable.

When Heat transport by Free convection occurs in a porous medium with a closed cavity the viscous dissipation is neglected. Aluminum foam samples of different pore sizes (5-60PPI) and porosities (0.8-0.99) were used to illustrate the effects of metal foam geometry on heat transfer (3).

\section{Conclusions and Suggestions}

This paper contains a novel model using feedback neural networks for a workpiece temperature prediction. The ANN model parameters of the result output were simulated using the ANN parameters The simulation was done using MATLAB $6.0^{\circledR}$ Neural Network Toolbox .The article aimed at presenting a calculation princeple based on statistical (ANN) and deterministic (CGM) models to evaluate the heat flux distribution generated by the flame gun in cylindrical workpiece. These models were complementary to each other, as the first one integrated the process variables that are not handled in the physical problem and the second one described by $q-r$ profiles required to predict the system response. The heat flux profiles exhibit a universal exponential decay characterized by increasing stability at a given radial position when increasing the preheating time. The ANN optimization revealed a two hidden layer structure which learned adequately the correlations (generalization property) encoded by the physical problem: the increase of heat flux with decrease of radial distance and with increase of preheating time. In addition, the ANN structure computed the heat flux decrease with increase of gunworkpiece distance

\section{References}

[1] P. Fauchius, A. Vandelle and B. Dussoubs, "Quo Vadis Thermal Spraying?” Journal of Thermal Spray Technology, Vol. 10, No. 1, 2001, pp. 44-66. doi:10.1361/105996301770349510

[2] Sampath and X. Jiang, "Substrate Temperature Spray Coatings," Materials Science and Engineering, Vol. 45, No. 124, 2002.

[3] I. Pop, "Modeling of Heat Flow in a Porous Cavity," International Journal of Heat and Mass Transfer, Vol. 23, No. 187, 2003.

[4] F. C. Lai, "Non Darcy Convection from a Line Source," International Communications in Heat and Mass Transfer, Vol. 12, No. 17, 1994, pp. 875-880.

[5] D. A. S. Ras and I. Pop, "Modeling of Natural Convection in Shallow Porous Concrete Slab to Control Heatflow," International Communications in Heat and Mass Transfer, Vol. 26, No. 6, 1999, pp. 761-770.

[6] D. A. S. Ras, "Free Convection Stagnation Point Flow in a Porous Media in a Thermal Nonequilibrium Region,” International Communications in Heat and Mass Transfer, Vol. 12, 1990, pp. 162-171.

[7] A. Postelneicu and I. Pop, "Convection in Porous Media with Generation," International Journal of Numerical Methods for Heat Transfer, Vol. 26, No. 8, 2002. 